Artículo

\title{
Potencial de cinco especies de leguminosas como abonos verdes en la zona altiplano de San Luis Potosí, México
}

\author{
Daniela Zavala-Sierra ${ }^{1}$ \\ Juan Carlos Rodríguez-Ortiz ${ }^{1 \S}$ \\ Jorge A. Alcalá-Jáuregui ${ }^{1}$ \\ Francisco H. Ruiz-Espinoza ${ }^{2}$ \\ Rigoberto González Mancillas ${ }^{3}$ \\ Humberto Rodríguez-Fuentes ${ }^{4}$ \\ Pablo Delgado Sánchez ${ }^{1}$
}

${ }^{1}$ Facultad de Agronomía y Veterinaria-UASLP. Palma de la Cruz, Soledad G. S. San Luis Potosí, México. ${ }^{2}$ Facultad de Agronomía-UABCS. México. ${ }^{3}$ Instituto Tecnológico de Torreón, Torreón, Coahuila, México.

${ }^{4}$ Facultad de Agronomía-UANL. Escobedo, Nuevo León.

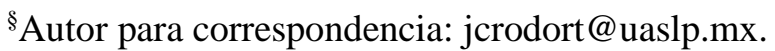

\section{Resumen}

Los abonos verdes (AV) son una alternativa para mejorar la fertilidad y conservación de suelos agrícolas en zonas semiáridas. Por tal motivo, se evaluó el potencial de cinco especies de leguminosas como AV en el altiplano de San Luis Potosí, México. Las especies de estudio fueron: 1) frijol dolichos negro (Lablab purpureos); 2) frijol dolichos café (Lablab purpureos); 3) frijol dolichos rojo (Lablab purpureos); 4) frijol yorimón (Vigna unguiculata); y 5) garbanzo (Cicer arietinum). El ensayo se realizó bajo un diseño de bloques al azar con tres repeticiones. En la etapa de floración de cada especie se realizó la evaluación. Las variables que se midieron fueron: biomasa, captura de carbono, huella de carbono, nitrógeno en planta y relación C/N. Los resultados indican que Dolichos rojo fue la más sobresaliente en producción de biomasa $(6.5 \mathrm{t}$ $\left.\mathrm{ha}^{-1}\right)$, captura de carbono $\left(2.97 \mathrm{t} \mathrm{ha}^{-1}\right)$ y huella de carbono $\left(-4.3 \mathrm{t} \mathrm{ha}^{-1}\right)$. Cicer arietinum sobresalió en concentrar $\mathrm{N}$ en sus tejidos $(2.03,1.47$ y $1.87 \%$ en parte aérea, raíz y planta completa) y podría aportar al suelo hasta $83 \mathrm{~kg} \mathrm{ha}^{-1} \mathrm{~N}$. Cicer sp., tuvo la más baja relación C/N con 24.12, con lo que tendría una mineralización más rápida que las demás especies. Como conclusión, las cinco especies tienen potencial para usarse como AV en la zona de estudio y son una alternativa importante para mejorar los suelos agrícolas y el medio ambiente.

Palabras clave: Lablab, Cicer, Vigna, carbono, nitrógeno.

Recibido: febrero de 2018

Aceptado: marzo de 2018 


\section{Introducción}

En México, como a nivel mundial, los suelos agrícolas se degradan como consecuencia de las diversas actividades humanas. Un análisis realizado estima que una pérdida mundial de $0.3 \%$ del rendimiento anual de los cultivos ocurre debido a la erosion. De continuar dichas tasas, se podría tener una reducción total de $10 \%$ del rendimiento en la producción de alimentos para el año 2050, lo que equivale a 150 millones de ha de producción de cultivos (FAO, 2016). En el estado San Luis Potosí, México, el mal uso de los suelos ha causado diferentes daños en sus propiedades, lo cual ha generado problemas en los rendimientos de los cultivos, además de perder fertilidad en cada ciclo (Martínez, 2014).

La alta energía requerida para la producción de fertilizantes, los altos costos de los mismos, la inexistencia de subsidios a los productos agrícolas y las limitaciones crediticias, en algunos casos, han despertado el interés en la búsqueda de alternativas tecnológicas más eficientes para el suministro de materia orgánica (MO) y nitrógeno en los cultivos (De la Cruz, 2015). En el caso de la energía requerida en la sintesis de fertilizantes, se estima un requerimiento de $64.65 \mathrm{MJ} \mathrm{kg}^{-1} \mathrm{~N}$ para la urea y de $52.62 \mathrm{MJ} \mathrm{kg}^{-1} \mathrm{~N}$ de otras fuentes de $\mathrm{N}$ (IDEA, 2007).

Una de estas alternativas, para mejorar la fertilidad y conservación de los suelos, consiste en la utilización de leguminosas en asociación y/o rotación como abonos verdes (AV), con el fin de aprovechar su potencial para la fijación de $\mathrm{N}_{2}$ atmosférico y al mismo tiempo el aporte de materia orgánica de rápida descomposición para mejorar la fertilidad del suelo (Prager et al., 2012). Este insumo natural ha sido reconocido por contribuir a la recuperación de la fertilidad y las características físicas del suelo, así como por su capacidad de controlar plagas, malezas y nemátodos, pero especialmente por su capacidad para incrementar la concentración de $\mathrm{MO}$ en el suelo (Beltrán et al., 2009). García et al. (2010) agrega que los AV contribuyen en el secuestro de carbono, que es de gran beneficio ambiental. Se estima que este elemento se ha perdido en los suelos a razón de $66 \pm 12$ billones de toneladas a nivel mundial desde el año 1850 (FAO, 2016).

Un primer paso, para el desarrollo y adopción de una tecnología agrícola, es la evaluación del desempeño de variedades en un ambiente dado, ya que es sabido que el comportamiento fisiológico y agronómico de un material vegetal está definido por la parte genética, el ambiente y la interacción entre ambos. Diversos estudios sobre evaluación de variedades de leguminosas para uso como abonos verdes en zonas áridas se hicieron en el estado de Baja California Sur con las especies del género Lablab (Beltrán et al., 2005 y 2009). Los autores concluyen que los suelos mejoraron significativamente en cuanto a sus propiedades físicas y químicas, y que las aportaciones de $\mathrm{N}$ al incorporarse los AV al suelo pueden alzanzar los $240 \mathrm{~kg} \mathrm{ha}^{-1}$. Sin embargo, en la zona semiárida del altiplano Potosino, donde se tienen más de 20 mil ha bajo sistemas hortícolas intensivos, no se ha reportado el uso de AV. Al respecto, García et al. (2010) señalan la necesidad de seguir investigando con especies con potencial para tolerar y ser productivos bajo condiciones adversas (zonas áridas y semiáridas).

El estudio tuvo como objetivo evaluar el potencial de cinco especies de leguminosas como abonos verdes en el altiplano potosino (San Luis Potosí, México) a través de la biomasa generada, captura de carbono, huella de carbono, nitrógeno y la relación $\mathrm{C} / \mathrm{N}$. 
El presente trabajo se realizó en el campus de la Facultad de Agronomía y Veterinaria, de la UASLP, ubicado en ejido Palma de la Cruz, municipio de Soledad de Graciano Sánchez, estado de

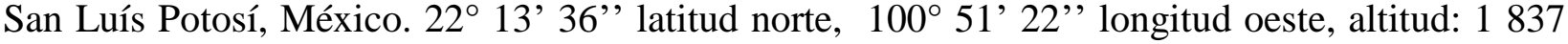
msnm. El clima es semiárido con temperatura media de $17.9{ }^{\circ} \mathrm{C}$ y precipitación de $341 \mathrm{~mm}$ año ${ }^{-1}$. El suelo presentó las siguientes características: textura, franco arenosa; $\mathrm{pH}, 7.6$, conductividad eléctrica, $2 \mathrm{dS} \mathrm{m}^{-1}$, materia orgánica, $1.1 \%$ (bajo) y nitrógeno inorgánico, $1.7 \mathrm{mg} \mathrm{kg}^{-1}$ (muy bajo).

Los materiales evaluados como abonos verdes fueron: 1) frijol dolichos negro (Lablab purpureus); 2) frijol dolichos café (Lablab purpureus); 3) frijol dolichos rojo (Lablab purpureus); 4) frijol yorimón (Vigna unguiculata); y 5) garbanzo de uso forrajero (Cicer arietinum). Las especies dolichos y yorimón, fueron elegidas por su adaptación y buen desempeño como AV en ambientes áridos, similares a la zona de estudio (Beltrán et al., 2005 y 2009). Por su parte, Cicer arietinum fue elegida por ser una especie que ha presentado buen crecimiento en las zonas centro y media del estado de San Luis Potosí, y por su condición de leguminosa. La busquedad de especies con potencial como AV es ampliamente recomendada (García et al., 2010).

Los AV se sembraron el día 13 de junio de 2013. La densidad de población fue de 5.5 plantas por $\mathrm{m}^{2}$. Las parcelas experimentales fueron de 3 surcos de $5 \mathrm{~m}$ de largo x $0.9 \mathrm{~m}$ de ancho cada una $\left(13.5 \mathrm{~m}^{2}\right)$. La distribución de los tratamientos fue a través de un diseño de bloques al azar con 3 repeticiones. En total se dispuso de una superficie de $202.5 \mathrm{~m}^{2}$. Las practicas al cultivo consistieron en dos deshierbes y escardas manuales durante los primeros 30 días, a partir de la siembra de los AV. Los riegos se realizaron para mantener una humedad del suelo disponible de entre $70 \%$ y $90 \%$

A los 115 días después de la siembra se realizó el muestreo de plantas, que fue el momento de inicio de la etapa fenológica de floración. Seis plantas completas representativas de la población fueron colectadas por tratamiento y repetición. En el laboratorio se lavaron las muestras con agua destilada y se procedió a la separación de la raíz de la parte aérea de cada planta. Posteriormente fueron puestas en bolsas de papel perforado y colocadas en horno de secado con aire forzado a 65 ${ }^{\circ} \mathrm{C}$ hasta obtener peso constante.

Las variables que se midieron como indicadoras del potencial de las leguminosas como abono verde en la zona altiplano de San Luis Potosí, México, fueron:

1) Biomasa, es una variable importante en la evaluación del crecimiento y producción de la planta, indican el potencial productivo de una especie en una zona determinada e integran su capacidad genética con la interacción con el medio ambiente (Ávila et al., 2010). Esta variable fue medida en función del peso seco de cada muestra, pesando de forma independiente la raíz de la parte aérea. Para obtener la biomasa por hectárea se multiplicó en resultado individual por la densidad

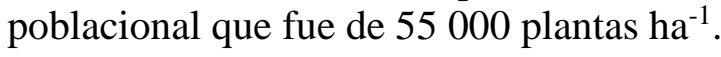

2) Carbono en biomasa, esta variable representa un valor de la eficiencia fotosíntetica de las plantas y es importante para el suelo ya que se relaciona con la sustentabilidad de los sistemas agrícolas afectando las propiedades del suelo relacionadas con el rendimiento sostenido de los 
cultivos. Fue estimado considerando que $45 \%$ del peso seco se compone de carbono en las especies vegetales (Azcón y Talón, 2008). De esta manera, la biomasa obtenida por hectárea se multiplicó por 0.45 .

3) Huella de carbono, significa la cantidad de $\mathrm{CO}_{2}$ capturado en la biomasa vegetal mas el $\mathrm{CO}_{2}$ equivalente que se liberaría en la fabricación de fertilizante nitrogenado (en este caso, nitrato amónico) en las cantidades de $\mathrm{N}$ que fueron fijadas por los $\mathrm{AV}$. $\mathrm{El} \mathrm{CO}_{2}$ capturado se estimó multiplicando el carbono en biomasa $\left(\mathrm{kg} \mathrm{ha}^{-1}\right)$ por 3.67 (factor de conversión de $\mathrm{C}$ a $\mathrm{CO}_{2}$, cociente que resulta de los pesos moleculares del dióxido de carbono 44 y del carbono 12). $\mathrm{El} \mathrm{CO}_{2}$ equivalente emitido a la atmósfera por $\mathrm{kg}$ de nitrógeno fijado en forma de fertilizantes y su transporte, se estimó con el valor $3.7 \mathrm{~kg}$ de $\mathrm{CO}_{2} \mathrm{~kg} \mathrm{~N}^{-1}$ (Yara, 2015).

4) Concentración de nitrógeno en planta, este es el elemento más limitante en la agricultura mundial, es fijado por los microorganismos del suelo en asociación con las leguminosas, como los AV en estudio. El nitrógeno en tejido vegetal se realizó a través del método Kjeldahl (Rodríguez y Rodríguez, 2002), y representa la cantidad de nitrógeno presente por biomasa obtenida. Este valor considera de manera proporcional el nitrógeno en la planta.

5) Nitrógeno por ha ${ }^{-1}\left(\mathrm{~kg} \mathrm{~N} \mathrm{ha}^{-1}\right)$, el contenido de nitrógeno por planta se multiplicó por la densidad de plantas por hectárea. Este valor considera el nitrógeno total absoluto fijado que se estaría incorporando al suelo.

6) Relación $\mathrm{C} / \mathrm{N}$, es útil para conocer la velocidad de descomposición de un AV e integrarse al suelo (Martínez et al., 2008). Fue determinada con el cociente de la división entre el carbono total y nitrogeno total estimado.

Para todas las variables en estudio se hizo análisis de varianza. En el caso de la variable porcentaje de $\mathrm{N}$ en la planta, previo al ANOVA, los valores se transformaron con la función arc sen $\sqrt{\text { porcentaje }}$, con la intención de normalizar los valores de porcentaje (Reyes, 1992). En las varibales donde se encontró diferencias significaticas se porcedio a hacer una comparación de medias con la prueba de Tukey $(p \leq 0.05)$. El software de diseños experimentales de la FAUANL se utilizó para analizar los datos obtenidos (Olivares, 2012).

Los resultados obtenidos en biomasa indican un buen crecimiento de las cinco especies en la parcela experimental de estudio (Figura 1a). Un estudio hechos en zonas aridas reportan resultados similares a los de este ensayo (Beltrán et al., 2009). En dicho estudio se reportan pesos secos por planta de 90 y 80 g por planta de los género Lablab y Vigna, respectivamente. En el presente ensayo, la especie más sobresaliente fue Lablab (frijol dolichos rojo), con pesos mayores a $109 \mathrm{~g}$ por planta $(p \leq 0.05)$, mientras que Vigna (frijol yorimón) obtuvo peso seco promedio por planta de $67.4 \mathrm{~g}$.

Beltrán et al. (2009) reportan haber tenido, con una densidad de población de 62500 plantas ha $^{-1}$, peso seco total de $5.6 \mathrm{t} \mathrm{ha}^{-1}$ de biomasa producida por Lablab. En este ensayo se tuvo una densidad de 55000 plantas, lo que da un peso seco total de $5.9 \mathrm{t} \mathrm{ha}^{-1}$ y $2.7 \mathrm{t} \mathrm{ha}^{-1}$ de carbono fijado (Figura 1b). También es relevante el haber obtenido pesos secos mayores a los encontrados en zonas tropicales como lo reporta Sosa et al. (2014), quienes obtuvieron rendimientos de parte aérea de 4 
t ha ${ }^{-1}$ con una asociación de Mucuna pruriensis L. var. Utilis con Zea mays var. ICA 305. El carbono fijado por las especies vegetales, que será incorporado al suelo, será indispensable para el crecimiento de plantas y el establecimiento de biota, ya que interviene en todos los procesos estructurales y metabólicos de los seres vivos (Lehmann y Kleber, 2015).

La ganancia ambiental de los AV se observa en la Figura 1c, donde el frijol dolichos rojo logró tener el mejor valor ambiental al estimarse una huella de carbono de $-4.3 \mathrm{t} \mathrm{ha}^{-1} \mathrm{CO}_{2}$ equivalente emitidos a la atmósfera (el signo negativo representa el $\mathrm{CO}_{2}$ capturado por $\mathrm{kg}$ de $\mathrm{N}$ en la biomasa) (Bentrup et al., 2004). Este valor comprende el ahorro de $\mathrm{CO}_{2}$ de la fabricación y transportar nitrógeno químico sintético en las unidades en que el $\mathrm{N}$ fue fijado por los $\mathrm{AV}$.

En cuanto a los resultados obtenidos en nitrógeno, sobresale de la especie Cicer arientnum (garbanzo), que logró concentrar $2.03 \%, 1.47 \%$ y $1.87 \%$ de $\mathrm{N}$ en parte aérea, raíz y total. En el caso de la raíz, fue superior estadísticamente al resto de las especies $(p \leq 0.05)$ (Figura 1d). En un estudio realizado en Oaxaca por Ruiz y Loaeza (2003), donde utilizaron las especies de trigo, soya y garbanzo, como AV, obtuvieron concentraciones de $\mathrm{N}$ en tejido vegetal de 1.73, 1.29 y 0.92 , respectivamente. Tales valores son similares a las especies estudiadas en este ensayo y, en el caso de garbanzo, la diferencia es del doble. Sin embargo, los resultados que se obtuvieron por las especies del género Lablab y Vigna son menores a los reportados por Beltrán et al. (2009) en estos mismos géneros. Las concentraciones de nitrógeno que encontraron en el tejido vegetal fueron, en promedio de más de $4 \%$.

Garate y Bonilla (2008), mencionan que los contenidos minerales entre las especies vegetales varían, incluso cuando las comparaciones se establecen utilizando los mismo órganos y de la misma edad fisiológica. Señalan varios factores determinantes: a) la dotación genética de la planta; b) la disponibilidad de nutrientes en el suelo o en el medio de cultivo; y c) el momento fenológico o edad de la planta y el órgano o tejido vegetal que se considere. Al respecto, consideramos que las diferencias encontradas en los contenidos de $\mathrm{N}$ en la planta, de este ensayo, son meramente genéticos, más que la disponibilidad de nutrientes, ya que las cinco especies crecieron en un suelo con mismas características químicas y nutrientes, así como el haberse cosechado en la misma etapa fenológica.

Los resultados en nitrógeno total, que puede ser incoporado al suelo, lo que depende de la concentración de $\mathrm{N}$ en los tejidos y la biomasa, la especie Cicer sp. vuelve a destacarse. Obtuvo el valor más elevado en toda la planta con $83 \mathrm{~kg} \mathrm{~N} \mathrm{ha}^{-1}$ de incorporarse la planta completa al suelo y $14.86 \mathrm{~kg} \mathrm{~N} \mathrm{ha}^{-1}$ en raíz, en este caso fue mayor al resto de las especies estadísticamnete $(p<0.05)$ (Figura 1e). Tales valores significan hasta $50 \%$ del nitrógeno necesario para un cultivo subsecuente, como pueden ser: lechuga, chile, calabaza, cilantro, ajo, cebolla y otros, que son cultivos de importancia económica en la zona del altiplano. Sin embargo, los $83 \mathrm{~kg} \mathrm{de} \mathrm{N} \mathrm{ha}^{-1}$, son mucho menos que los $240 \mathrm{~kg}$ de $\mathrm{N}^{-1}$ que reportan haber obtenido Beltrán et al. (2009) con el género Lablab en Baja California Sur. Las diferencias pueden deberse a las elevadas concentraciones de $\mathrm{N}$ en tejido vegetal que ellos obtuvieron, como se mencionó anteriormente (> 4\%), así como las elevadas densidades de población (62 500 plantas ha $^{-1}$ ).

Los resultado en la relación $\mathrm{C} / \mathrm{N}$ indican que el garbanzo (Cicer arietinum) es la especie que tendría más facilidad de incorporar el nitrógeno de sus tejidos al suelo y ser arpovechado por los cultivos subsecuentes. Los valores obtenidos son los mas bajos del grupo de las especies en estudio (relación 
< 25 en planta completa) (Figura 1f). Según Martin y Rivera (2000) las plantas con relación C/N menor de 25, la mineralización del $\mathrm{N}$ es rápida, mientras que las plantas con relación $\mathrm{C} / \mathrm{N}$ mayores de 25 forman una cobertura estable, que contribuye al incremento del contenido de materia orgánica y por ende a mejorar la estructura del suelo y a protegerlo del impacto de la lluvia y la radiación solar; además, favorece el desarrollo del sistema radical, la formación de nódulos y la fijación simbiótica del nitrógeno.
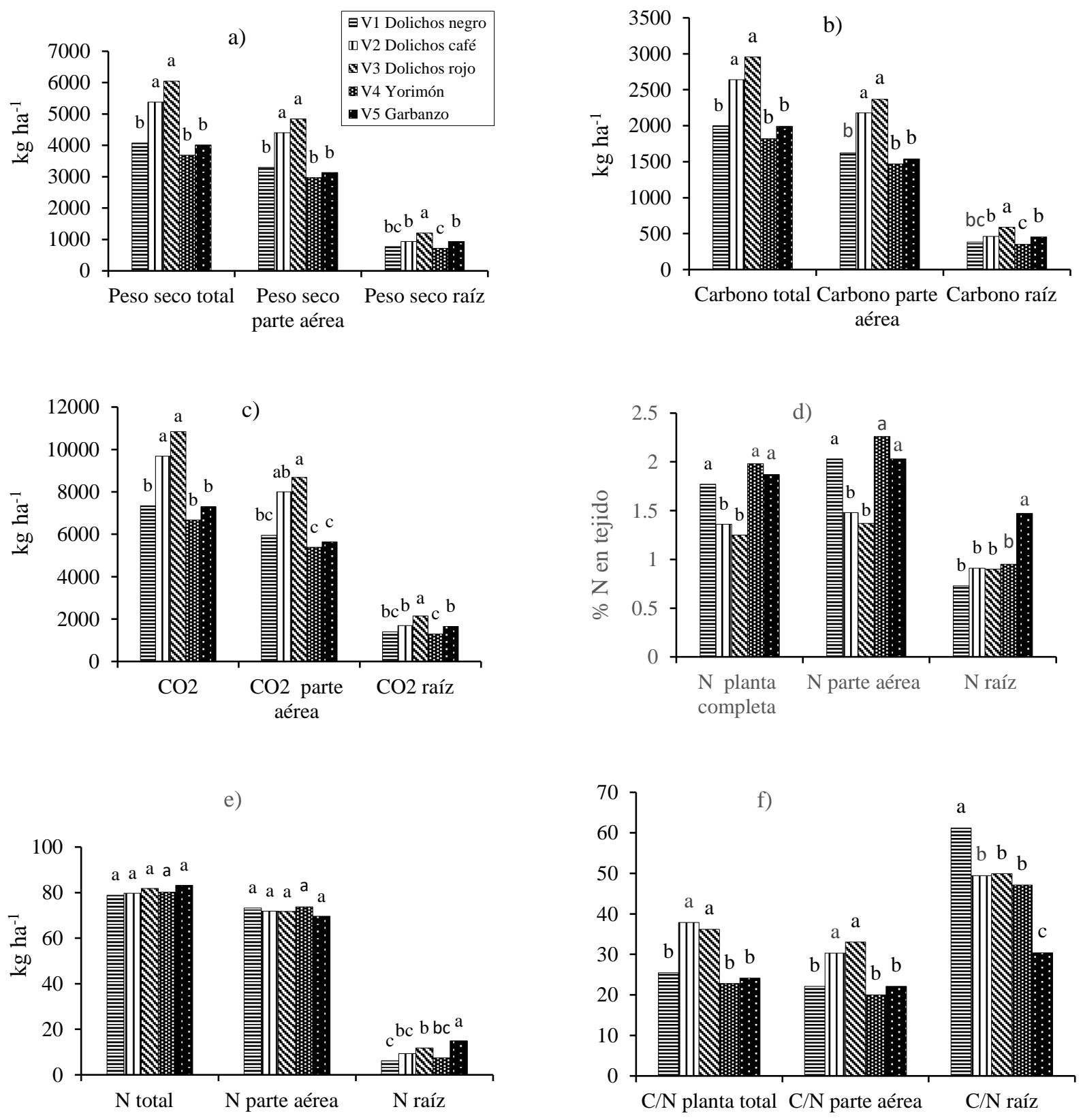

Figura 1. Figura 1. a) Peso seco de los cinco AV. b) Carbono en los cinco AV. c) Huella de carbono en los cinco AV $\left(\mathrm{CO}_{2}\right.$ capturado en los $\left.\mathrm{AV}\right)$. d) Nitrógeno en tejido vegetal en los cinco AV (\%). e) Nitrógeno (N) en tejido vegetal que se aportaría al suelo de los cinco AV. f) Relación C/N de los cinco AV. Medias seguidas con misma letra en cada barra indican que son estadísticamente 


\section{Conclusiones}

Las cinco especies del estudio: frijol dolichos negro (Lablab purpureos), frijol dolichos café (Lablab purpureos), frijol dolichos rojo (Lablab purpureos), frijol yorimón (Vigna unguiculata) y garbanzo (Cicer arietinum), tienen potencial para usarse como abonos verdes en la zona de altiplano de San Luis Potosí, México, y son una alternativa importante para mejorar los suelos agrícolas y medio ambiente.

La especie de frijol dolichos rojo fue la más sobresaliente en producción de biomasa $\left(6.5 \mathrm{t} \mathrm{ha}^{-1}\right)$, captura de carbono (2.97 $\left.\mathrm{t} \mathrm{ha}^{-1}\right)$ y huella de carbono (-4.3 $\left.\mathrm{t} \mathrm{ha}^{-1}\right)$. La especie Cicer arietinum sobresalió por concentrar $\mathrm{N}$ en sus tejidos $(2.03,1.47$ y $1.87 \%$ en parte aérea, raíz y total, respectivamente), con lo que podría aportar al suelo hasta $83 \mathrm{~kg} \mathrm{~N} \mathrm{ha}^{-1}$. También obtuvo la relación $\mathrm{C} / \mathrm{N}$ más baja (24.14), con lo que tendría una mineralización más rápida que las demás especies del estudio.

\section{Literatura citada}

Ávila, N. Y.; Murillo B.; Palacios, E.; De Luna, A. y García, J. L. 2010. Modelos de predicción del rendimiento de grano y caracterización de cinco cultivares de frijol Yorimón. Trop. Subtrop. Agroecosys. 12(1):11-18.

Azcon, B. J. y Talon, M. 2008. Fundamentos de la fisiología vegetal. $2^{\text {nd }}$ Edición. Ed. McGrawHill. $631 \mathrm{p}$.

Beltrán, A.; García, J. L.; Valdés, R.; Murillo, B.; Troyo, E.; Larrinaga, J.; Ruiz, H.; Fenech, L. y García, R. 2005. Sistemas de labranza, incorporación de abono verde y recuperación de la fertilidad de un yermosol háplico. Terra Latinoam. 23:381-387.

Beltrán, A.; García, J. L.; Ruiz, H.; Fenech, L.; Murillo, B.; Palacios, E. and Troyo, E. 2009. Nutrimental potential of red dolichos, brown dolichos and cowpea for green manure produced under three tillage Systems. Trop. Subtrop. Agroecosys. 10:487-495.

Brentrup, F.; Küsters, J.; Lammel, J.; Barraclough, P. and Kuhlmann, H. 2004. Investigation of the environmental impact of agricultural crop production using the life cycle assessment (LCA) methodology. Part II: application of the LCA methodology to investigate the environmental impact of different $\mathrm{N}$ fertilizer rates in cereal production. Europ. J. Agron. 20:265-279.

De la Cruz, C. C. A. 2015. Contribución de abonos verdes en la producción sostenible de caña de azúcar (Saccharum Officinarum L.), en la hacienda la floresta del municipio BugalagrandeValle del Cauca. Universidad Nacional Abierta y a Distancia. Escuela de Ciencias Agrícolas, Pecuarias y del Medio Ambiente. Palmira, Colombia. 61 pp.

Gárate, A. y Bonilla, I. 2008. Nutrición mineral y producción vegetal. In: fundamentos de fisiología vegetal. Azcón-Bieto J. y Talon, M. (Coords.). 2 ${ }^{\text {nd }}$ (Ed.). Ed. McGrawHill. España. 143-144 pp.

García, J. L.; Murillo, B.; Nieto, A.; Fortis; M.; Márquez, C.; Castellanos, E.; Quiñones, J. y Ávila, N. 2010. Avances en investigación y perspectivas del aprovechamiento de los abonos verdes en la agricultura. Terra Latinoam. 28(4):391-399.

García, J. L.; Orona, I.; Preciado. P.; Flores, A.; Murillo, B. and Troyo, E. 2010. Nutrients use efficiency in legume crops to climatic changes. In: Yadav, S.; McNeil, D.; Redden, R. and Patil, S. (Eds.). Climatic change and management of cool season grain legume crops. Springer Dordrecht Heidelberg, London, New York. 193-206 pp. 
IDEA. 2007. Instituto para la Diversificacion y Ahorro de la Energía. Ahorro, eficiencia energética y fertilizacion nitrogenada. Ministerio de industria, turismo y comercio. España. 46 pp.

Lehmann, J. and Kleber, M. 2015. The contentious nature of soil organic matter. Nature. 528:60-68.

Martín, G. M. y Rivera, R. 2000. Mineralización de dos especies promisorias de abonos verdes en suelo ferralítico rojo (ferralsol) mediante el método de incubación aeróbica. Cultivos Tropicales. 21(2):73-78.

Martínez, E.; Fuentes, J. and Acevedo, H. 2008. Soil organic carbon and soil properties. J. Soil Sc. Plant Nutr. 8(1):68-96.

Martínez, G. M. 2014. Efecto acumulado de la agricultura de conservación en la producción de maíz elotero de riego en el altiplano de San Luis Potosí. Programa de investigación: fertilidad de suelo y nutrición vegetal. INIFAP, San Luis Potosí. 2 p.

Olivares, S. E. 2012. Paquete de diseños experimentales. FAUANL. Versión 1.1. Facultad de Agronomía, Universidad Autónoma de Nuevo León. Marín, N. L. México.

FAO. 2016. Organización de las Naciones Unidas para la Alimentación y la Agricultura (FAO). Estado Mundial del Recurso Suelo. Resumen técnico. Roma, Italia.

Prager, M.; Sanclemente, E.; Sánchez de Prager, M; Gallego, J. y Ángel, D. 2012. Abonos verdes: tecnología para el manejo agroecológico de los cultivos. Rev. Agroecología. Murcia, España. 7:53- 62.

Reyes-Castañeda, R. 1992. Diseños de experimentos aplicados. Ed. Trillas, 302-309 pp.

Rodríguez, H. y Rodríguez, J. 2002. Métodos de análisis de suelos y plantas. Criterios de interpretación. Trillas primera edición, México.145-168 pp.

Ruiz, J. y Loaeza, G. 2003. Evaluacion de abonos verdes en asociasion con maíz de temporal en los Valles Centrales de Oaxaca, Mexico. Terrra Latinoam. 21(3):409-415.

Sosa, A.; Sanclamente, E. y Sánchez de Prager, M. 2014. Influencia de abonos verdes sobre la dinámica de nitrógeno en un Typic Hapluster del Valle del Cauca. Colombia. Acta Agron. 63(4):343-351.

Yara International. 2015. La huella de carbono de los fertilizantes. http://www.yara.com.mx/cropnutrition/medio-ambiente/reduciendo-huella-de-carbono/reducir-la-huella-de-carbono/. 